\title{
Calcium Deficiency Rickets
}

National Cancer Institute

\section{Source}

National Cancer Institute. Calcium Deficiency Rickets. NCI Thesaurus. Code C131421.

Rickets due to low calcium concentrations, the cause of which can be nutritional or genetic. 